\title{
Dietetic approach in patient with dehydration and electrolyte imbalance caused by diarrhea - a case report
}

\author{
Dragana Mladenovska*, Dafina Boshkoska, Elena Karabeleski, Menka Andreska, \\ Suzana Atanasovikj, Bojana Janeku, Aleksandra Kapedanovska Nestorovska, \\ Tanja Petreska Ivanovska, Kristina Mladenovska, Lidija Petrushevska-Tozi
}

Faculty of Pharmacy, Ss. Cyril and Methodius University, Mother Theresa No. 47, 1000, Skopje, N. Macedonia

\section{Introduction}

Acute diarrhea is defined as three or more episodes of partially formed or watery stool per day, which lasts less than 14 days. Diarrheal illness accounts for approximately 2.5 million deaths per year worldwide (de Bruyn., 2010). Bacterial and viral infections account for most episodes of acute diarrhea in adults, with viruses being the most common infectious cause in the community. Electrolyte imbalance as a result of acute diarrhea is common and frequently unrecognized resulting in morbidity and mortality. Therefore, effective replacement of electrolytes in diarrheal patients is necessary based on exact knowledge of changes in the composition of body fluids. Early recognition of the acute diarrhea and analysis of common electrolyte abnormalities is necessary to provide correction. The first line of treatment should include solutions for oral rehydration and adequate nutritional support that will prevent electrolyte imbalance from progressing (Casburn-Jones and Farthing, 2004). Based on a patient case, this paper aims to stress the importance of nutritional support and the role of certified dietitian in preventing severe consequences from acute diarrhea.

*mladenovska.dragana@gmail.com

\section{Case presentation}

Eighteen-year-old male, recently returned from a two-week trip to Middle East country was feeling ill for several days, which started the day before arriving home. He reported 5 to 10 episodes of diarrhea each day, which was resolved after taking loperamide. Fecal smear indicated gross blood with leukocytes. Admitting diagnosis was moderate dehydration with $\mathrm{R} / \mathrm{O}$ bacterial vs. viral gastroenteritis.

After physical assessment, the following parameters were evidenced: general appearancelethargic 18-year-old male; vitals- temperature $38.6{ }^{\circ} \mathrm{C}$; blood pressure 80/65; heart rate $89 \mathrm{BPM}$; respiratory rate $22 \mathrm{BPM}$; heart- moderately elevated pulse; eyes- sunken, sclera clear without evidence of tears; ears- clear; nose- dry mucous membranes; throat- dry mucous membranes, no inflammation; genitalia- unremarkable; neurologic- alert, oriented, irritable; extremities- no joint deformity or muscle tenderness, no edema; skin- warm, dry; reduced capillary refill (approximately 2 seconds); chest/lungs- clear to auscultation and percussion; abdomen- tender, nondistended, minimal bowel sounds.

After nutritional assessment, the following anthropometric measurements were reported: height$188 \mathrm{~cm}$; weight $81 \mathrm{~kg}$; BMI $22.9 \mathrm{~kg} / \mathrm{m}^{2}$. After 
biochemical testing the following data were obtained: total protein $7.2 \mathrm{~g} / \mathrm{dL}$, albumin $4.9 \mathrm{~g} / \mathrm{dL}$, sodium $154 \mathrm{mmol} / \mathrm{L}$, potassium $3.2 \mathrm{mmol} / \mathrm{L}$, chlorides $107 \mathrm{mmol} / \mathrm{L}$, phosphates $4.0 \mathrm{mmol} / \mathrm{L}$, BUN $21 \mathrm{mg} / \mathrm{dL}$, chromium $1.4 \mathrm{mg} / \mathrm{dL}, \mathrm{Hgb} 15.5$ $\mathrm{g} / \mathrm{dL}$, Hct $41 \%$, WBC $17 \times 10^{3} / \mathrm{mm}^{3}$.

\section{Discussion}

The goals of the nutritional intervention in acute diarrhea are to restore lost fluids, normalize water, electrolytes and acid-base balance, reduction of gastrointestinal motility and initiation of normal stool formation. Re-colonization of the gastrointestinal tract should be also established. With a proper diet regimen, the patient returns to a normal diet without worsening symptoms (Nelms, 2010).

Based on the patient data, nutritional diagnosis included: (P) inadequate fluid and food intake related to (E) increased losses of fluids and fever as evidenced by (S) diarrhea, hypernatremia, and elevated serum chloride ions.

The patient had confirmed mild dehydration and the first step of nutritional intervention was to administer oral rehydration therapy. The fluid requirements were calculated based on the body weight, being $2835-3240 \mathrm{~mL}(30-35 \mathrm{ml} / \mathrm{kg} \times 81 \mathrm{~kg})$. For the best outcome, a low osmolality oral rehydration solution (ORS) $(240-250 \mathrm{mOsm} / \mathrm{L})$ was prescribed, knowing that high osmolality ORS cause additional water absorption in the gastrointestinal tract and may worsen the condition and diarrhea.

Nutrition therapy was designed to prevent the consumption of beverages containing large amounts of simple carbohydrates (lactose, sucrose, and fructose), sugar alcohols (sorbitol, xylitol, and mannitol), caffeine and non-alcoholic foods fibers (cellulose). The patient was advised to consume foods containing soluble fiber and pectin (to promote stool formation). As best starters, green bananas that contain a lot of pectin, toast with white bread or boiled rice were recommended. Other foods were also slowly introduced to provide a sufficient variety of nutrients. The patient was advised not to consume spicy and hot foods as well as sodas because they can cause additional irritation in the gastrointestinal tract (GIT).
Another mandatory step in the treatment of diarrhea is administration of probiotics and prebiotics in order to re-colonize GIT and restore normal function and nutrient absorption. Foods that naturally contain prebiotics and probiotics were recommended to be consumed more often.

Daily menus were created and at the beginning of therapy meals were small but frequent, usually every 3-4 hours, with several interruptions when the diarrhea returned, to determine which of the foods worsened the condition. The patient was advised to drink minimum of $2.8 \mathrm{~L}$ of water during the day (water, unsweetened tea or rehydration drinks; the amount of yogurt was counted in daily fluids).

To evaluate the outcome of the nutrition therapy, the following parameters were regularly monitored and recorded: serum electrolytes, weight, food and fluid intake, food tolerance, frequency and amount of diarrhea.

\section{Conclusion}

Adequate nutritional support plays critical role in restoring normal function of GIT and preventing future complications of acute diarrhea. For this to be achieved, the diarrhea dietitian can help discover how different foods impact the patient's digestion and what triggers the symptoms. He/she can point to the adequate food items and solutions for proper hydration; food supplements for restoring balance in the GIT and in addition, helps in modifying lifestyle habits.

\section{References}

de Bruyn, G., 2008. Diarrhoea in adults (acute). BMJ Clin. Evid. 0901.

Casburn-Jones, A.C., Farthing M.J.G., 2004. Management of infectious diarrhoea. Gut 53, 236-305.

Nelms, M.N., 2010. Diseases of the lower gastrointestinal tract, second ed., in: Cossio, Y. (Ed.), Nutrition Therapy \& Pathophysiology. Cengage Learning, Belmont, pp. 376-436. 